Miller RH. Am J Dis Child 1963;106:197).

\title{
CEREBELLITIS AND ACUTE HYDROCEPHALUS
}

A case of obstructive hydrocephalus and bilateral cerebellitis with swelling in a 14-year-old boy is reported from the Department of Child Health, St George's Hospital, and the Departments of Neurosurgery and Neuroradiology, Atkinson Morley's Hospital, London, UK. The child presented with a 2-month history of recurrent headache and a 2-week history of occipital headache, nausea and vomiting, without signs of acute infection or immunization. Fundi showed bilateral papilledema. Apart from mild dysdiadochokinesia, the neurologic exam was otherwise normal. MRI showed obstructive hydrocephalus and marked bilateral cerebellar swelling with increased signal on T-2 weighted imaging, compatible with acute inflammation. Clinical and MRI signs resolved in 4 weeks following treatment with corticosteroids, and the neurologic exam was normal at 12 month follow-up. (Aylett SE, O'Neill KS, De Sousa C, Britton J. Cerebellitis presenting as acute hydrocephalus. Child's Nerv Syst March 1998;14:139-141). (Respond: Dr SE Aylett, The Wolfson Centre, Mecklenburgh Square, London WC1N 2AP, UK).

COMMENT. The clinical and MRI findings and recovery following steroids were considered consistent with a parainflammatory cerebellitis, although viral studies were negative. Prompt diagnosis and therapy are important because of the danger of tonsillar herniation.

\section{ATTENTION DEFICIT DISORDERS}

\section{CEREBELLAR VERMIS ANOMALY IN ADHD}

Cerebellar and vermal volumes were measured by quantitative MRI studies in 46 right-handed boys with attention deficit hyperactivity disorder (ADHD) and 47 matched healthy controls, and results were analyzed at the Child Psychiatry Branch, National Institute of Mental Health, Bethesda, MD. Both area and volumetric analyses showed a smaller cerebellar vermis in boys with ADHD, and the posterior inferior lobules VIII to X were particularly involved. Total cerebral volume was $6 \%$ smaller in the ADHD group, also. WISC-R vocabulary and block design scores were significantly lower in ADHD compared to control children. The findings were not related to prenatal alcohol exposure nor to methylphenidate treatment. A cerebello-thalamo-prefrontal circuit dysfunction is postulated in ADHD. (Berquin PC, Giedd JN, Jacobsen LK et al. Cerebellum in attention-deficit hyperactivity disorder. A morphometric MRI study. Neurology April 1998;50:10871093). (Reprints: Dr FX Castellanos, 10 Center Drive, Room 3N202, Bethesda, MD 20892).

COMMENT. The cerebellar vermis is smaller in right-handed boys with $\mathrm{ADHD}$, and the reduction in volume involves particularly the posterior inferior lobules VIII to $\mathrm{X}$. The smaller cerebral volumes might explain the significantly lower IQ scores in ADHD children compared to controls. Previous studies by Dr Castellanos at the NIH showed that ADHD was associated with loss of normal asymmetry in the caudate nucleus, smaller right anterior frontal area, and smaller cerebellum, and IQ scores correlated with total cerebral volume. Researchers at the Masssachusetts General Hospital, Boston, have reported a smaller splenium of the corpus callosum in ADHD children, and the Johns Hopkins group of investigators found a smaller globus pallidus volume (see Progress in Pediatric Neurology III, 1997;p 212). These studies add to the growing volume of data supporting a structural brain pathology as the basis for ADHD (Millichap JG. 